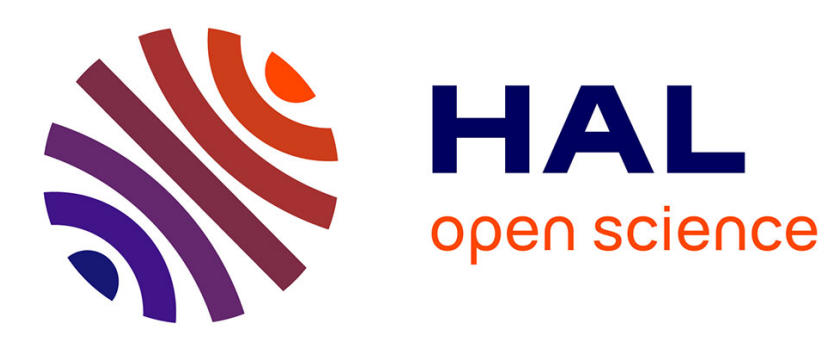

\title{
Experimental analysis of submicrometer optical intensity distributions after an opaque disk
}

\author{
O. Emile, Janine Emile
}

\section{To cite this version:}

O. Emile, Janine Emile. Experimental analysis of submicrometer optical intensity distributions after an opaque disk. Applied optics, 2020, 59 (6), pp.1678-1683. 10.1364/AO.387699 . hal-02535642

HAL Id: hal-02535642

https://hal-univ-rennes1.archives-ouvertes.fr/hal-02535642

Submitted on 17 Apr 2020

HAL is a multi-disciplinary open access archive for the deposit and dissemination of scientific research documents, whether they are published or not. The documents may come from teaching and research institutions in France or abroad, or from public or private research centers.
L'archive ouverte pluridisciplinaire HAL, est destinée au dépôt et à la diffusion de documents scientifiques de niveau recherche, publiés ou non, émanant des établissements d'enseignement et de recherche français ou étrangers, des laboratoires publics ou privés. 


\title{
Experimental analysis of sub-micrometer optical intensity distributions after an opaque disk
}

\author{
Olivier EMILE ${ }^{1, *}$ AND JANINE EMILE ${ }^{2}$ \\ ${ }^{1}$ Université Rennes 1, F-35000 Rennes, France \\ ${ }^{2}$ Université Rennes 1, CNRS, IPR (Institut de Physique de Rennes) - UMR 6251, F-35000 Rennes, France \\ *Corresponding author: emile@univ-rennes1.fr
}

Compiled January 14, 2020

\begin{abstract}
Generation of sub-wavelength beam sizes is a fascinating challenge with several implications. The observation of a $120 \mathrm{~nm}$ laser spot size in the visible part of the spectrum is here reported. It has a size variation of less than $10 \%$ on a distance of $50 \mu \mathrm{m}$ along the axis of propagation. This so-called Arago spot results from the diffraction of the light from a laser diode by the edges of an absorbing disk. Applications are discussed. Furthermore, hollow beams carrying orbital angular momentum with $400 \mathrm{~nm}$ diameter dark spot in the centre are evidenced. This paves the way towards atom lithography via atom guiding or new spectroscopy on forbidden transitions. (- 2020 Optical Society of America
\end{abstract}

http://dx.doi.org/10.1364/ao.XX.XXXXXX

\section{INTRODUCTION}

On the one hand, an increasing number of experiments and optical devices requires concentrated light beams on scales shorter than the wavelength $\lambda$. Prominent examples could be encountered in optical lithography, confocal microscopy, optical data storage or particle trapping. Several technics have been developed to bypass the fundamental diffraction limit [1,2], that is of the order of a fraction of $\lambda$, such as fluorescence nanoscopy [3], metamaterials [4], near-field and plasmonic [5, 6], two-photon laser writing $[7,8]$, or non-diffracting beams $[9,10]$. However, because of fundamental limitations or technical restrictions, the beam size is usually larger than a few hundred nanometers.

On the other hand, the so-called Arago spot consists of a bright-diffracted spot at the center of the shadow of a circular object that is uniformly illuminated [11,12]. At the beginning of the $19^{\text {th }}$ century, it played a major role in the demonstration of the wave nature of light $[13,14]$. It is also responsible for damages in unstable cavities [15], and has been shown to propagate faster than light [16-19]. This superluminal behavior is due to the fact that the Arago spot could be considered as an azimuthal superposition of plane waves of equal inclination in respect to the optical axis. Then, as for scissor blades, their contact point can move faster than light [20]. But most of all, it propagates in a quasi non-diffracting way and its size should decrease while approaching the disk. One may then wonder whether such a decrease could be experimentally demonstrated, and what is the ultimate limit. The aim of this article is thus to investigate the properties of the Arago spot close to the occulting object.

\section{THEORETICAL CONSIDERATIONS}

As the central part of a collimated laser beam is occulted by a disk centered on the beam axis (see figure 1a), a bright Arago spot appears in the shadow of the disk. Its intensity distribution can be calculated using the Huygens-Fresnel principle [21]. Every unobstructed point of a wavefront becomes the source of a secondary spherical wavelet and the amplitude of the optical field at a given point is the superposition of all the secondary wavelets with their relative phases. Such numerical calculations have been carried out and appear in red in figure 1c. The radial intensity distribution $I(r)$ at a distance $z$ from the occulting disk can also be approximated, in the near field diffraction regime, by a squared zeroth Bessel function $J_{0}$ [22].

$$
I(r) \simeq I_{0} \frac{z^{2}}{z^{2}+(d / 2)^{2}} J_{0}^{2}\left(\frac{\pi r}{\lambda} \frac{d}{\left(z^{2}+(d / 2)^{2}\right)^{1 / 2}}\right)
$$

where $d$ is the disk diameter (see figure $1 \mathrm{a}$ ), and $I_{0}$ is the intensity of the incoming beam, considering the incoming beam as a plane wave. As $z$ tends towards zero, the Bessel function equals $J_{0}^{2}(2 \pi r / \lambda)$. It doesn't depend on the disk diameter any more. Note that, far from the disk, Eq. 1 reduces to the usual formula $[13,14,23]$,

$$
I(r) \simeq I_{0} \frac{z^{2}}{z^{2}+(d / 2)^{2}} J_{0}^{2}\left(\frac{\pi r d}{\lambda z}\right)
$$

It has been recently shown that light diffraction from an occulting disk could lead to a dark Arago spot instead of a bright one. The light beam has to be a vortex beam [24] instead of a plane wave. Such vortex beams carry orbital angular momentum and have a non-uniform phase distribution. On a plane 


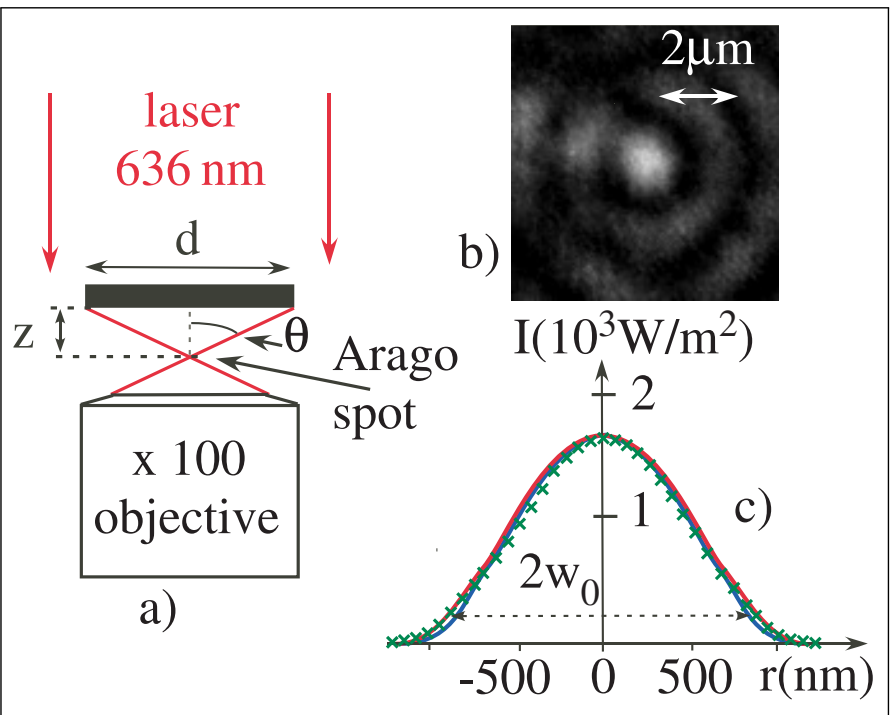

Fig. 1. Set-up. (a) Light of a red collimated laser diode that impinges on an occulting disk $d=600 \mu \mathrm{m}$. The diffracted light interferes in the shadow of the disk (at the intersection of the red lines). The spot is observed with a microscope with a x100 objective and a monochrome gray-scale camera. (b). Picture of the Arago spot at a distance of $1.4 \mathrm{~mm}$ from the disk. Scale: $2 \mu \mathrm{m}$. (c). Intensity distribution corresponding to the pixel intensity, together with a fit according to equation 1 (blue), and a numerical estimation of the intensity distribution using the Huygens-Fresnel principle (red). $w_{0}$ : spot waist.

perpendicular to the direction of propagation, the phase varies uniformly from 0 to $2 \pi \ell$. $\ell$ is an integer number called the topological charge of the beam. When diffracted by a disk centered on the beam axis, the spot conserves its topological charge, and its intensity distribution like for Eq. 1 is described by the square of the $\ell^{\text {th }}$ Bessel function $J_{\ell}$. It varies as

$$
I(r) \simeq I_{0} \frac{z^{2}}{z^{2}+(d / 2)^{2}} J_{\ell}^{2}\left(\frac{\pi r}{\lambda} \frac{d}{\left(z^{2}+(d / 2)^{2}\right)^{1 / 2}}\right)
$$

Like for Eq. 2, far from the disk the $J_{\ell}$ function could be simplified to $J_{\ell}^{2}(\pi r d /(\lambda z))$ [25-27].

\section{EXPERIMENTAL SET-UP}

A collimated laser beam $(\lambda=636,532,488$ and $404 \mathrm{~nm}$, Oxyus lasers L6Cc) impinges on a dark absorbing object (see figure 1a). Since light is transported by an optical fiber, it is non-polarized. In order not to saturate the detection, its power is limited to 2 $\mathrm{mW}$ for the red, green and blue lasers and to $6 \mathrm{~mW}$ for the violet laser. An aspheric collimator lens (Thorlabs CFC-2X-A) adjusts the laser beam size to the the disk size. The dark absorbing disks (diameter 500 to $900 \mu \mathrm{m}$ ) are made of chromium deposited on a $2 \mathrm{~mm}$ thick BK7 glass. The roughness of the contour of the chromium disk is less then $0.3 \mu \mathrm{m}$. This has been verified with a microscope. According to Eq. 1, with $z=100 \mu \mathrm{m}$, and $d=275$ $\mu \mathrm{m}$, this corresponds to a variation of the spot size of the order of $0.1 \mathrm{~nm}$, and a phase variation of less than a fraction of $2 \pi$. Integrated over the whole disk, this could thus be neglected. Actually, our chromium deposited disks are "class 4 " bought from Photo Data. The roughness is rather in the $50 \mathrm{~nm}$ range. Special care is taken to align the axis of the beam with the axes of the disk and the microscope. The chromium deposited side points towards the objective of the microscope, so that the Arago spot travels in air only.

The diffracted light interferes in a bright spot in the centre of the shadow of the disk. It propagates and it is imaged with a microscope. The microscope is an inverted Leica microscope (Leica DMi8) with a x100 objective (NA 0.95 in air). The numerical aperture (NA) is defined as $n \sin \theta, n$ being the index in the medium we look at (air in our case), and $\theta$ being the maximal angle of incidence. The light rays diffracted by the edges of the disk, impinge with an angle $\theta$ regarded to the vertical axis that equals $\theta=\tan ^{-1}(d / 2 z)$ at the spot location (see figure 1a). It thus means that we cannot observe a spot at a distance shorter than $100 \mu \mathrm{m}$ for a $d=600 \mu \mathrm{m}$ disk.

The position of the disk can be adjusted thanks to servocontrolled micro-stages positioners in the XYZ directions, with sub-micrometer resolution. The position $z=0$ corresponds to a focus of the microscope on the chromium deposited dark disk. The verification of the $z=0$ position is performed every time at the beginning and at the end of each experiment. The camera is a monochrome gray-scale Hamamatsu CCD camera (C11440). We have calibrated the imaging system against a millimeter sight. One pixel corresponds to $60 \mathrm{~nm}$. The recorded images are analyzed using ImageJ software [28]. We first identify the Arago's spot at a distance of $z=4 \mathrm{~mm}$ as the initial setting position, before reducing the distance $z$.

\section{RESULTS}

An example of an image of a spot is displayed in figure $1 \mathrm{~b}$. It is located at a distance $z=1.40 \mathrm{~mm}$ from a $d=600 \mu \mathrm{m}$ disk. The bright spot roughly corresponds to 30 pixels $(1.8 \mu \mathrm{m})$. The variation with the azimuthal direction is mainly due to diffuse light from all the collection optics (including the lenses), and from multiple reflections at interfaces. Figure 1c shows the experimental intensity and a fit using equation 1, together with a numerical estimation of the intensity according to the Huygens-Fresnel principle. The agreement is very good. It also validates the approximations made to evaluate the diffracted spot of equation 1 . Note also that the only adjustable parameter is the incident light intensity. We characterize the spot by its waist, as for Gaussian beams [15]. It is defined as the distance from the spot axis at which the irradiance has fallen to $1 / e^{2}$ of its maximum value. It corresponds to the value of the square of the Bessel function for 1.75. In figure $1, w_{0}$ equals $0.84 \mu \mathrm{m}$.

Although the value $w_{0}=0.84 \mu \mathrm{m}$ is already of the order of the light wavelength $(\lambda=0.636 \mu \mathrm{m})$, one may wonder whether smaller sizes could be obtained. We have thus varied the distance $z$ between the observations and the disk. The resulting spot size variation is shown on figure $2 \mathrm{a}$. As expected from equation 1, and from numerical estimations, this variation is linear with $z$, far from the occulting disk. It then should tends towards $1.75 \lambda / \pi=166 \mathrm{~nm}$, for $\lambda=636 \mathrm{~nm}$. It is difficult to define a depth of focus of the Arago's spot, as for focused beams, since it doesn't result from a focusing. Nevertheless, the spot size variation is less than $10 \%$ over a $50 \mu \mathrm{m}$ distance in a region where the size is in the tenth of micrometer range. The smallest waist $w_{0}$ we measured, is $220 \pm 15 \mathrm{~nm}$, at a distance of $z=150$ $\mu \mathrm{m}$ from the absorbing disk. This is well below the wavelength. Since the beam size depends linearly on the wavelength, we then use several laser beams at $\lambda=532,488$ and $404 \mathrm{~nm}$ to decrease its size further.

As can be seen in figure $2 a$, the spot size varies linearly with 


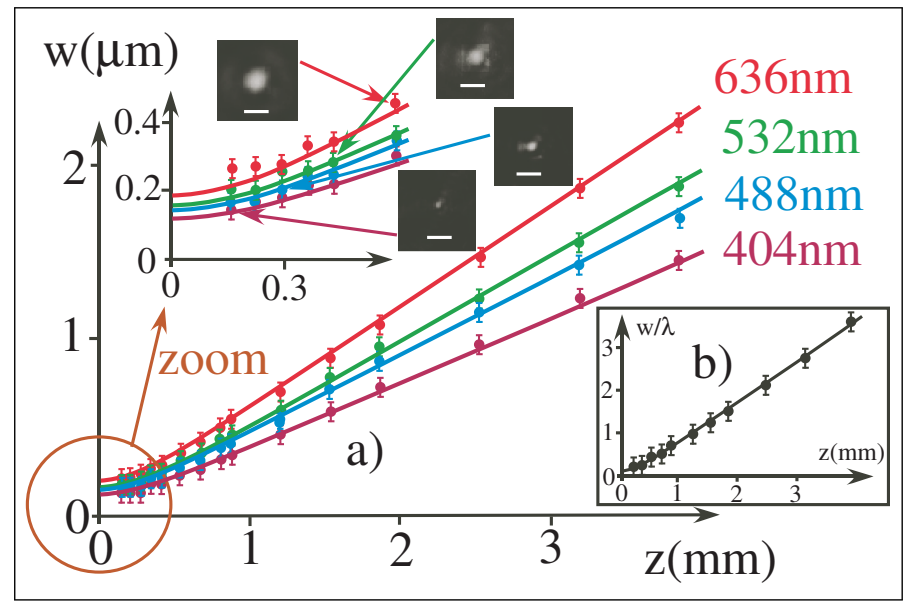

Fig. 2. a) Experimental spot size variation versus $z$ for 4 different laser wavelengths. Circles: experimental measurements. The error bars result from the resolution of the microscope and from uncertainties in the measurements. Solid lines: plots of equation 1, with no adjustable parameters. Inserts: pictures of spots for different wavelengths and different spot sizes. The white scale corresponds to $1 \mu \mathrm{m}$ and is the same for every picture. b) Same as a), but the experimental spot size $w$ has been normalized by $\lambda$. The experimental measurements for the 4 wavelengths end up on the same theoretical curve corresponding to equation 1.

the wavelength far from the occulting disk, as expected. Close to the disk its value is finite. The smallest spot we measure has a waist of $w_{0}=120 \pm 15 \mathrm{~nm}$ for the violet light, at a distance $z=150 \mu \mathrm{m}$. The ultimate theoretical value is $110 \mathrm{~nm}$. The intensity of the bright spot also limits its observation. According to equation 1 , the intensity should tend toward zero when approaching the disk. It is worth noting that one can plot for the 4 wavelengths the spot waist $w$ normalized by $\lambda$ (see $2 \mathrm{~b}$ ). Then, the experimental points are aligned on the same theoretical curve corresponding to equation 1 .

Besides, according to equation 2, the spot size is proportional to $\lambda z / d$ whereas the beam size of a Gaussian beam varies as $\lambda z / w_{0}$ [15]. There is thus a factor corresponding to $d / w_{0}$ between the divergence of a focused Gaussian beam and the linear variation of the spot we observed. For a strongly focused beam $\left(w_{0} \sim 1 \mu \mathrm{m}\right)$ the ratio is of the order of $10^{3}$. The Arago-spot is not limited by diffraction.

\section{DISCUSSION}

Alternatively, such spots could also be obtained using axicon lenses $[9,29]$. These elements are of conical shape instead of spherical one for usual lenses. As a Gaussian beam impinges on it, Snell's laws lead to an intensity distribution depending on $r$, that varies as a square Bessel function. In that case, the spot size is constant and imposed by the lens (angle of the cone $\alpha$ ). The intensity distribution scales with $J_{0}^{2}(2 \pi r(n-1) \tan \alpha / \lambda)[30,31]$. For an optical index $n$ of 1.5, the highest value of $\alpha$ is around $40^{\circ}$, leading to $\tan \alpha \sim 0.84$, and the Bessel spot holds on a very small distance. In the case of the Arago spot, the corresponding quantity goes to 1 and the Arago spot holds on a longer distance.

There are other technics that could also lead to small optical beam sizes. For example, so-called super oscillations have also been shown to enable sub-wavelength beam sizes [32]. However the beam size is of a fraction of the wavelength and holds for a few micrometers only, whereas Arago spots are in the hundreds nanometer range and hold for hundred of micrometers. Similar spot sizes have been also evidenced behind a disk of smaller size and on much smaller scale $[19,33]$, leading to a strong phase anomaly. This kind of spot also appears in photonic nanojets that originate from diffraction [34].

Apparently there may be a kind of paradox in our experiment: The spot originates from the diffraction of light by the edge of a disk. This diffracted light interferes to form a bright spot. However, its size is not limited by diffraction. This spot could be smaller then the wavelength and may beat the resolving power or the Rayleigh criterion of a usual microscope. Actually, this criterion tells us that a faithful image needs that all the spectra contribute to the formation of the image [13]. This is never possible using ordinary lenses. However, in the case of the Arago spot, all the spectra components are within a cone which angle is $\theta=\tan ^{-1}(d / 2 z)$. The azimuthal superposition of these waves leads to the Arago spot. As soon as the numerical aperture of the microscope is higher than this angle, all the components enter the microscope. There is no component left. It could then be that the image reach hundred of nanometer sizes, well below $\lambda$. It is very similar, in its principle, to negative refraction which has the power to focus all Fourier components of a 2D image making thus a perfect lens [35], also beating the Rayleigh criterion.

It is worth noting that either equation 1 or the HuygensFresnel principle relies on a scalar theory of diffraction. It becomes questionable when the angle between the incident and the diffracted spot tends toward $90^{\circ}$. A vectorial calculation could be needed. However, the agreement between calculations and experimental observations is very good. Probably, the scalar theory is here valid because the polarization of light is not linear. Besides, as noted in calculations about photonic nanojets, and in Bessel beams [33,34], the diffraction spot, in case of a linear polarization, would become slightly elliptic. The size of the spot in the direction perpendicular to the electric field is reduced. This would marginally affect the spot size. Anyway, we have added a linear polarizer at the end of the fiber and we didn't experimentally notice any difference.

Since this technics only requires a laser and an absorbing disk, it is easy to implement and it could find straightforward applications in many domains such as optical lithography, confocal microscopy or optical data storage. In the first case, a smaller light spot would lead to a much more defined tool that could be of valuable help in material processing [36]. Besides, there is very little constrain on the "depth of focus". In the later case, the size of the writing or reading beam is the main limitation for an increase of the storage capacity [37]. A decrease of the size spot by a factor of ten as shown here, would lead to an increase of the data storage capacity of one hundred.

Applications in particle trapping could also be considered. Actually, the intensity gradient in a plane perpendicular to the direction of propagation can be very steep. It could lead to very efficient dipole forces and to the trapping of particles. Arago spots with very small sizes could also be obtained for matter waves or even in other domains of the electromagnetic spectrum as for X-rays for example reaching sub-nanometer dimensions. It may be optimized for applications using $\mathrm{X}$-rays at grazing incidence. 


\section{GENERALIZATION TO LIGHT ORBITAL ANGULAR MOMENTUM (OAM)}

In order to generate spots carrying OAM, we have modified the experimental setup of figure 1 and replaced the fundamental beam illuminating the disk by a vortex beam with a topological charge $\ell=4$ at $\lambda=636 \mathrm{~nm}$. The vortex beams is generated using a spiral phase plate [38] (RC photonics). Like for the usual Arago spot, the centre of the vortex beam is aligned with the centre of the disk and with the axis of the microscope. The focus of the laser diode is adjusted so that the size of the vortex beam corresponds to the size of the disk. We can generate vortex beams with $\ell=1$ up to $\ell=8$ at $\lambda=636 \mathrm{~nm}$. The vortex nature of the beam is probed using a Young double slit experiment technics [39]. The choice of a $\ell=4$ beam results from a compromise. The realization of small dark spots needs to decreases $\ell$ whereas sharp edges need higher $\ell$.

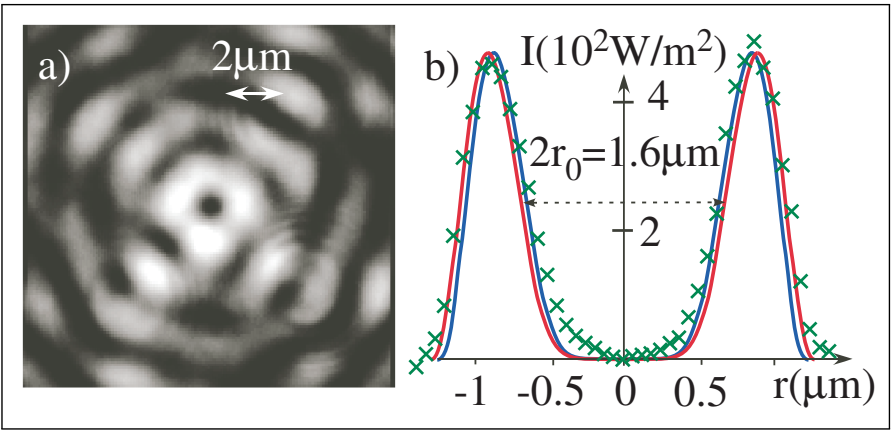

Fig. 3. Example of a $\ell=4$ diffracted beam. a) Photograph of the dark spot at a distance of $z=0.57 \mathrm{~mm}$, exemplifying the hollow in the middle of the light intensity distribution. b) Intensity distribution (the crosses correspond to the intensity of the pixels), together with a fit using the equation 3 (blue), and a numerical estimation of the intensity distribution using the Huygens-Fresnel principle (red).

An example of a diffracted beam at a distance $z=0.57 \mathrm{~mm}$ from the disk $(d=600 \mu \mathrm{m})$ is displayed on figure 3 . The light intensity distribution clearly corresponds to a circular hollow beam with a dark spot in its centre. The intensity distribution is well reproduced by numerical estimations using the HuygensFresnel principle (see figure $3 \mathrm{~b}$ ). It also matches a square $4^{\text {th }}$ order Bessel function (see equation 3). The small discrepancy between the fit and the experimental value, especially in the centre of the distribution, which is the most useful part, may result from the impurity of the beam (residual contribution from $\ell=1$ and $\ell=2$ modes). This is probably also the reason for the discrepancy in the wing of the distribution. For the sake of simplicity, we define the diameter of the dark spot at half width at half maximum, leading to a value of $0.8 \mu \mathrm{m}$.

We have also varied the distance between the disk and the spot (see figure 4). We find a linear variation of the dark spot diameter versus $z$, far from the occulting disk, in good agreement with equation 3 . The smallest dark spot diameter is of the order of $420 \mathrm{~nm}$ at $\lambda=636 \mathrm{~nm}$. The theoretical limit of the dark Arago spot diameter is $400 \mathrm{~nm}$, according to equation 3 .

Such hollow beams could have interesting consequences [40]. For example hollow beams have been proposed to guide atoms $[41,42]$. When the light frequency is adjusted so that the dipole electric force is repulsive, the atoms are forced to travel in the central part of the beam. However, getting sizes below the mi-

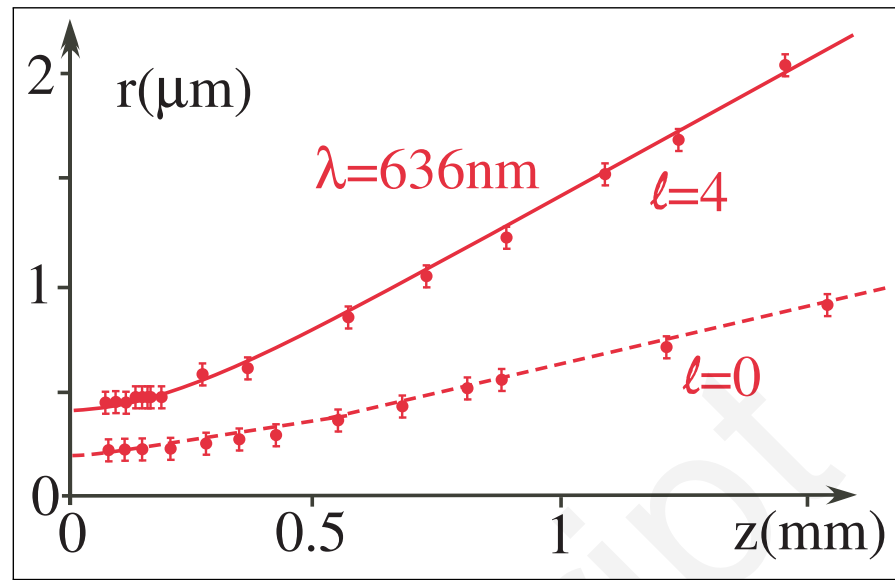

Fig. 4. Spot size variation versus $z$ for a $\ell=4$ diffracted beam. Circular points: experimental measurements. Error bars result from the microscope resolution, and for measurements uncertainties. Red solid line: plot from equation 3 , with no adjustable parameters. The dotted line and the experimental points correspond to the waist of the $\ell=0$ spot of figure 2, that have been added here for comparison.

crometer range is one of the present limitation of focused hollow beams. Of course, Arago spots are less efficient than usual hollow beams, or hollow guide fibers [43], to guide atoms on long distances. In the case of an Arago dark spot, the beam size increases significantly after $100 \mu \mathrm{m}$ propagation. Nevertheless, it could act as a funnel at the end of such guiding structures with extremely small dimensions, below the micrometer range. Those atoms could then be loaded in atomic traps, or used for lithography or to generate well collimated matter waves. Submicrometer particles could also be trapped in the dark region and rotated since vortex beams carry OAM [44]. Small size hollow beams have also been produced using nanophotonics. For example, shaping an azimuthally polarized wave with a planar binary phase lens leads to hollow spots with half width at half maximum of $0.6 \lambda[45,46]$. Hollow beams have also been reported from metamaterial structures [47]. Though, such hollow spots are either diffraction limited or rather difficult to realize [48].

However, one of the most stunning foreseen issues may be in a new kind of spectroscopy aimed at investigating forbidden transitions $[49,50]$ on atoms or molecules. Indeed, vortex beams could induce multipolar transitions due to selection rules corresponding to high values of $\ell$. There is no limitation to its value. Nevertheless, the vortex beam must then be of the order of the atomic size. Interactions with a Bose Einstein condensate [51] have been proposed in order to increase the spread of the atomic wave function.

It has also been proposed and demonstrated [52] to observe quadrupolar transitions using combinations of $\ell=1$ orbital angular momentum and spin angular momentum. Yet, the size of the optical beam that carries orbital angular momentum is still one of the main limitations of such beams, as for hollow beams. Actually, it is difficult to achieve small sizes using conventional focused vortex beams, because of the diffraction limit. Nevertheless, as described here, Arago dark spots could in principle be in the hundreds of nanometer range, getting closer to the atom typical size. Multipolar transitions even with $\ell>1$ may become possible and be likely observed. This would thus pave the way to a new spectroscopy. 


\section{CONCLUSION}

We have thus investigated the properties of the Arago spot close to an occulting disk. Its intensity distribution is described by a Bessel function. We have shown that $120 \mathrm{~nm}$ size spot could be obtained at a distance of $150 \mu \mathrm{m}$ behind the object, in good agreement with theoretical estimations. However, theoretically, it tends to a finite value close to our experimental value when approaching the disk. Nevertheless, such small size spots may pave the way towards new potentialities of Bessel beams [53]. In particular, applications in optical lithography or in data storage capacities have been discussed. Besides, it has been shown that nanometer size dark vortex beams can be achieved, with applications including atom guiding or forbidden transition spectroscopy.

Acknowledgments: The authors thank Renaud MATHEVET (LNCMI, University of Toulouse) and Pierre CHAVEL (Institut Charles FABRY, University of Paris XI Orsay) for fruitful preliminary discussions, and Hervé TABUTEAU (IPR, University of Rennes 1) for valuable remarks and suggestions. The laser was funded by the Contrat Plan Etat Région CPER BUFFON.

Disclosures. The authors declare no conflicts of interest.

\section{REFERENCES}

1. E. Betzig, J. K. Trautman, T. D. Harris, J. S. Weiner, and R. L Kostelak, "Breaking the diffraction barrier: optical microscopy on a nanometric scale," Science 251, 1468-1470 (1991).

2. H. Wang, C. J. Sheppard, K. Ravi, S. T. Ho, and G. Vienne, "Fighting against diffraction: apodization and near field diffraction structures," Laser Photon. Rev. 6, 354-392 (2012).

3. S. W. Hell, "Far-field optical nanoscopy," Science 316, 1153-1158 (2007).

4. P. V. Parimi, W. T. Lu, P. Vodo, and S. Sridhar, "Photonic crystals: Imaging by flat lens using negative refraction," Nature 426, 404 (2003).

5. W. Adams, M. Sadatgol, and D. Ö. Güney, "Review of near-field optics and superlenses for sub-diffraction-limited nano-imaging," AIP Advances 6, 100701 (2016).

6. K. A. Willets, A. J. Wilson, V. Sundaresan, and P. B. Joshi, "Superresolution imaging and plasmonics," Chem. Rev. 117, 7538-7582 (2017).

7. T. Gissibl, S. Thiele, A. Herkommer, and H. Giessen, "Two-photon direct laser writing of ultracompact multi-lens objectives," Nat. Photon. 10, 554-560 (2016).

8. G. Williams, M. Hunt, B. Boehm, A. May, M. Taverne, D. Ho, S. Giblin, D. Read, J. Rarity, R. Allenspach, and S. Ladak, "Two-photon lithography for 3D magnetic nanostructure fabrication," Nano Res. 11, 845-854 (2018).

9. F. Courvoisier, J. Zhang, M. K. Bhuyan, M. Jacquot, and J. M. Dudley, "Applications of femtosecond Bessel beams," Appl. Phys. A 112, 29-34 (2013).

10. J. Fu, Y. Wang, and P. Chen, "Generation of sub-half-wavelength nondiffracting beams with tunable depth of focus by focusing azimuthally polarized beams," Appl. Phys. B 123, 214 (2017).

11. R. E. English and N. George, "Diffraction patterns in the shadows of disks and obstacles," Appl. Opt. 27, 1581-1587 (1988).

12. G. E. Sommargren and H. J. Weaver, "Diffraction of light by an opaque sphere. 1: Description and properties of the diffraction pattern," Appl. Opt. 29, 4646-4657 (1990).

13. M. Born, E. Wolf: Principle of Optics, 7th edition (Cambridge University Press, Cambridge, 1999).

14. E. Hecht: Optics, 4th edition (Addison-Wesley, San Fransisco, 2001).

15. A. E. Siegman: Lasers (University Science Books, Mill Valley, 1990).

16. D. Chauvat, O. Emile, M. Brunel, and A. Le Floch, "Direct measurement of the central fringe velocity in Young-type experiments," Phys. Lett. A 295, 78-80 (2002).

17. D. Chauvat, O. Emile, M. Brunel, and A. Le Floch, "Huygens' principle and Young's experiment in the propagation of light beams," Am. J. Phys. 71, 1196-1198 (2003).

18. P. Piksarv, P. Bowlan, M. Lõhmus, M., H. Valtna-Lukner, R. Trebino, and P. Saari, "Diffraction of ultrashort Gaussian pulses within the framework of boundary diffraction wave theory," J. Opt. 14, 015701 (2012).

19. M. S. Kim, T. Scharf, C. Etrich, C. Rockstuhl, and H. H. Peter, "Longitudinal-differential interferometry: Direct imaging of axial superluminal phase propagation," Opt. Lett. 37, 305-307 (2012).

20. H. Valtna-Lukner, P. Bowlan, M. Lõhmus, P. Piksarv, R. Trebino, and P. Saari, "Direct spatiotemporal measurements of accelerating ultrashort Bessel-type light bullets," Opt. Express, 17, 14948-14955 (2009).

21. A. Sommerfeld: Lectures on Theoretical Physics: Optics Vol. IV. (Academic Press INC, 1954).

22. Z. L. Horváth and Z. Bor, "Diffraction of short pulses with boundary diffraction wave theory," Phys. Rev. E 63, 026601 (2001).

23. J. E. Harvey and J. L. Forgham, "The spot of Arago: New relevance for an old phenomenon," Am. J. Phys. 52, 243-247 (1984).

24. S. M. Barnett, M. Babiker, and M. J. Padgett, "Optical orbital angular momentum," Philos. Trans. A Math. Phys. Eng. Sci. 375, 20150444 (2017).

25. P. Fischer, S. E. Skelton, C. G. Leburn, C. T. Streuber, E. M. Wright, and K. Dholakia, "The dark spots of Arago," Opt. Express 15, 11860-11873 (2007).

26. O. Emile, A. Voisin, R. Niemiec, B. Viaris de Lesegno, L. Pruvost, G. Ropars, J. Emile, and C. Brousseau, "Dark zone in the centre of the Arago-Poisson diffraction spot of a helical laser beam," EPL 101, 54005 (2013).

27. D. Hebri, S Rasouli, and A. M. Dezfouli, "Theory of diffraction of vortex beams from structured apertures and generation of elegant elliptical vortex Hermite-Gaussian beams, " J. Opt. Soc. Am. A 36, 839-852 (2019).

28. C. A. Schneider, W. S. Rasband, and K. W Eliceiri, "NIH Image to ImageJ: 25 years of image analysis," Nat. Methods 9, 671-675 (2012)

29. F. O. Fahrbach, P. Simon, and A. Rohrbach, "Microscopy with selfreconstructing beams," Nat. Photon. 4, 780-785 (2010).

30. G. Roy and R. Tremblay, "Influence of the divergence of a laser beam on the axial intensity distribution of an axicon." Opt. Commun. 34 1-3 (1980).

31. S. K. Tiwari, S. R. Mishra, S. P. Ram, and H. S. Rawat, "Generation of a Bessel beam of variable spot size." Appl. Opt. 51 3718-3725 (2012).

32. G. H. Yuan, E. T. Rogers, and N. I. Zheludev, "Achromatic superoscillatory lenses with sub-wavelength focusing," Light Sci. Appl. 6, e17036 (2017).

33. M. S. Kim, T. Scharf, A. da Costa Assafrao, C. Rockstuhl, S. F. Pereira, H. P. Urbach, and H. P. Herzig, "Phase anomalies in Bessel-Gauss beams." Opt. Express 20, 28929-28940 (2012).

34. A. Heifetz, S. C. Kong, A. V. Sahakian, A. Taflove, and V. Backman, "Photonic nanojets," J. Comput. Theor. Nanosci. 6, 1979-1992 (2009).

35. J. B. Pendry, "Negative refraction makes a perfect lens, " Phys. Rev. Lett. 85, 3966-3968 (2000).

36. M. Duocastella and C. B. Arnold, "Bessel and annular beams for materials processing," Laser Photon. Rev. 6, 607-621 (2012).

37. E. N. Glezer, M. Milosavljevic, L. Huang, R. J. Finlay, T. H. Her, J. P. Callan, and E. Mazur, "Three-dimensional optical storage inside transparent materials," Opt. Lett. 21, 2023-2025 (1996).

38. M. W. Beijersbergen, R. P. C. Coerwinkel, M. Kristensen, and J. P. Woerdman, "Helical-wavefront laser beams produced with a spiral phaseplate," Opt. Commun. 112, 321-327 (1994).

39. O. Emile and J. Emile, "Young's double-slit interference pattern from a twisted beam," Appl. Phys. B 117, 487-491 (2014).

40. J. Yin, W. Gao, and Y. Zhu, "Generation of dark hollow beams and their applications," Progress in Optics 45, 119-204 (2003).

41. Y. Song, D. Milam, and W. T. Hill, "Long, narrow all-light atom guide," Opt. Lett. 24, 1805-1807 (1999).

42. V. Carrat, C. Cabrera-Gutiérrez, M. Jacquey, J. W. Tabosa, B. Viaris 
de Lesegno, and L. Pruvost, "Long-distance channeling of cold atoms exiting a 2D magneto-optical trap by a Laguerre-Gaussian laser beam," Opt. Lett. 39, 719-722 (2014).

43. M. Xin, W. S. Leong, Z. Chen, and S. Y. Lan, "An atom interferometer inside a hollow-core photonic crystal fiber," Sci. Adv. 4, e1701723 (2018).

44. O. Emile, C. Brousseau, J. Emile, R. Niemiec, K. Madhjoubi, and B. Thidé, "Electromagnetically induced torque on a large ring in the microwave range," Phys. Rev. Lett. 112, 053902 (2014).

45. X. Li, M. Pu, Z. Zhao, X. Ma, J. Jin, Y., Wang, P. Gao, and X. Luo, "Catenary nanostructures as compact Bessel beam generators." Sci. Rep. 6, 20524 (2016).

46. Z. Wu, Q. Jin, S. Zhang, K. Zhang, L. Wang, L. Dai, Z. Wen, Z. Zhang, G. Liang, Y. Liu, and G. Chen, "Generating a three-dimensional hollow spot with sub-diffraction transverse size by a focused cylindrical vector wave." Opt. Express 26, 7866-7875 (2018).

47. Z. Yao, Z.SU, and Y. Wang, "Graphene-based hyperbolic metamaterials for a tunable subwavelength dark hollow beam" Appl. Opt. 58, 82578261 (2019).

48. G. Chen, Z. Q. Wen, and C. W. Qiu, "Superoscillation: from physics to optical applications." Light Sci. 8, 1-13 (2019).

49. R. F. Pettifer, S. P. Collins, and D. Laundy, "Quadrupole transitions revealed by Borrmann spectroscopy," Nature 454, 196-199 (2008).

50. M. Babiker, D. L. Andrews, and V. E. Lembessis, "Atoms in complex twisted light," J. Opt. 21, 013001 (2019).

51. P. K. Mondal, B. Deb, and S. Majumder, "Angular momentum transfer in interaction of Laguerre-Gaussian beams with atoms and molecules," Phys. Rev. A 89, 063418 (2014).

52. C. T. Schmiegelow, J. Schulz, H. Kaufmann, T. Ruster, U. G. Poschinger, and F. Schmidt-Kaler, "Transfer of optical orbital angular momentum to a bound electron," Nat. Commun. 7, 12998 (2016).

53. D. McGloin, and K. Dholakia, "Bessel beams: diffraction in a new light," Contemp. Phys. 46, 15-28 (2005). 